OPEN ACCESS

Edited by:

Yao Lin,

Fujian University of Traditional Chinese

Medicine, China

Reviewed by:

Qingshui Wang,

Fujian Normal University, China

Weilin Liu,

Fujian University of Traditional Chinese

Medicine, China

${ }^{*}$ Correspondence:

Xiaojuan Hong

365358649@qq.com

Rongjiang Jin

cdzyydxirj@126.com

Juan Li

785939016@qq.com

${ }^{\dagger}$ These authors have contributed equally to this work and share first

authorship

Specialty section: This article was submitted to

Molecular Diagnostics and

Therapeutics,

a section of the journal

Frontiers in Molecular Biosciences

Received: 19 October 2021

Accepted: 21 December 2021

Published: 13 January 2022

Citation:

Zhong D, Li Y, Huang Y, Hong X, Li J and Jin $R$ (2022) Molecular Mechanisms of Exercise on Cancer: A Bibliometrics Study and Visualization

Analysis via CiteSpace.

Front. Mol. Biosci. 8:797902.

doi: 10.3389/fmolb.2021.797902

\section{Molecular Mechanisms of Exercise on Cancer: A Bibliometrics Study and Visualization Analysis via CiteSpace}

\author{
Dongling Zhong ${ }^{1 \dagger}$, Yuxi $\mathrm{Li}^{2 \dagger}$, Yijie Huang ${ }^{3 \dagger}$, Xiaojuan Hong ${ }^{2 *}$, Juan $\mathrm{Li}^{1 *}$ and Rongjiang Jin ${ }^{1 *}$ \\ ${ }^{1}$ School of Health Preservation and Rehabilitation, Chengdu University of Traditional Chinese Medicine, Chengdu, China, ${ }^{2}$ School \\ of Acupuncture Moxibustion and Tuina, The Third Affiliated Hospital, Chengdu University of Traditional Chinese Medicine, \\ Chengdu, China, ${ }^{3}$ The Seventh Affiliated Hospital, Sun Yat-sen University, Shenzhen, China
}

Objective: To analyze the research hot spots and frontiers of molecular mechanisms of exercise on cancer via CiteSpace.

Method: Related publications in the Web of Science Core Collection Science Citation Index Expanded were retrieved from inception to November 27th, 2021. Then we used CiteSpace to generate network maps and identify top authors, institutions, countries, keywords, co-cited authors, journals, references and research trends.

Results: A total of 1,130 related publications were retrieved. The most productive author and journal were Lee W Jones and PLOS ONE. Hanahan D and Warburg O were the most cited authors. Fudan University and Shanghai Jiao Tong University were the leading institutions, while China was the leading country. Top-cited authors and references generally focused on the epidemiology and hallmarks of cancer. Top five keywords with both high frequency and high betweenness centrality were breast cancer, aerobic glycolysis, oxidative stress, gene expression, skeletal muscle. Keyword "warburg effect" ranked first with the highest citation burst, while "inflammation", "hepatocellular carcinoma", "epithelial mesenchymal transition", and "adipose tissue" were emerging research foci.

Conclusion: This study analyzed the research hot spots and frontiers of molecular mechanisms of exercise on cancer via CiteSpace. Based on the results, altered metabolism (aerobic glycolysis, insulin resistance, myokines), oxidative stress, gene expression and apoptosis were hot-research mechanisms of exercise on cancer. Emerging research foci of mechanisms were generally around inflammation, epithelial mesenchymal transition and adipokines. In addition, future studies could carry in-depth research of interactions between different mechanisms and try to elucidate the recommended doses and intensities of exercise for cancer, especially in breast, colorectal, prostate cancer and hepatocellular carcinoma.

Keywords: exercise, cancer, molecular mechanisms, bibliometrics, visualization analysis, citespace 


\section{INTRODUCTION}

Cancer is one of the leading causes of disability and mortality worldwide. According to latest estimates of the International Agency for Research on Cancer (IARC) (https://www.iarc.who. int/), there were about 19.3 million new cases of cancer, 10 million cancer deaths worldwide in 2020. In 2040, there will be 28.4 million new cancer cases.

Exercise plays an essential role in the management of cancer, especially in cancer prevention, cancer progression control, cancer-related outcomes improvement (Galvao et al., 2010; Speck et al., 2010; Magne et al., 2011). According to a study involving 430,000 people, leisure-time physical activity was associated with lower risks of several cancer types (Moore et al., 2016). In addition, exercise could reduce cancer risk factors such as obesity (Swift et al., 2018), inflammation (Metsios et al., 2020) and improve health-related outcomes in cancer survivors (Campbell et al., 2019). On the contrary, physical inactivity could increase the risk of colon cancer (Wolin et al., 2009) and breast cancer (Kyu et al., 2016). Therefore, multiple organizations including the American Cancer Society (ACS) (Rock et al., 2012), the American College of Sports Medicine (ACSM) (Schmitz et al., 2010), Exercise and Sports Science Australia (ESSA) (Hayes et al., 2019) have published exercise guidelines for cancer survivors.

Due to the rapid development of technologies, the possible molecular mechanisms of exercise are being illuminated, which may be possibly related to changes in the serum markers level, inflammation markers, oxidative stress and so on (Hojman et al., 2018). However, research hot spots and frontiers of this field remain unclear.

CiteSpace is a Java-based application to analyze and visualize the hot spots and research frontiers in the scientific literature of a discipline or knowledge domain in a certain period with metrology, co-occurrence analysis and cluster analysis (Chen, 2004; Chen, 2006). In this study, we intended to analyze the hot spots and research frontiers of molecular mechanisms of exercise on cancer via CiteSpace, which may help us understand the curative and preventive effects of exercise for cancer better.

\section{METHODS}

\section{Search Strategy}

We retrieved articles in the Web of Science Core Collection Science Citation Index Expanded (SCI-Expanded) from inception to November 27th, 2021, using the following terms: exercise, neoplasms and molecular mechanism. Table 1 shows the detailed search strategy.

\section{Inclusion and Exclusion Criteria}

Peer-reviewed published original articles or reviews about the molecular mechanisms of exercise on cancer were included.

Exclusion criteria were: 1) conference abstracts or corrigendum documents; 2) unpublished articles; 3) repeated publications; 4) unrelated articles.

\section{Bibliometrics and Visualization Analysis}

We exported retrieved articles in plain text format with full records and references, named "download_XXX.txt" and then imported into CiteSpace 5.8.R3 for further analysis. When mapping visualization knowledge figures, we followed the main procedural steps of CiteSpace, including time slicing, thresholding, modeling, pruning, merging, and mapping (Chen, 2004). Central concepts of CiteSpace includes burst detection, betweenness centrality, and heterogeneous networks, which can help to timely visualize the research status, hot spots, and frontiers (Chen, 2006). Nodes in different maps represent authors, institutions, countries or keywords. Size of nodes indicates the frequency of occurrence or citation, and color of nodes indicated the occurrence or citation years. Besides, nodes with purple trims suggests high betweenness centrality, which are often identified as hot spots or turning points in a field.

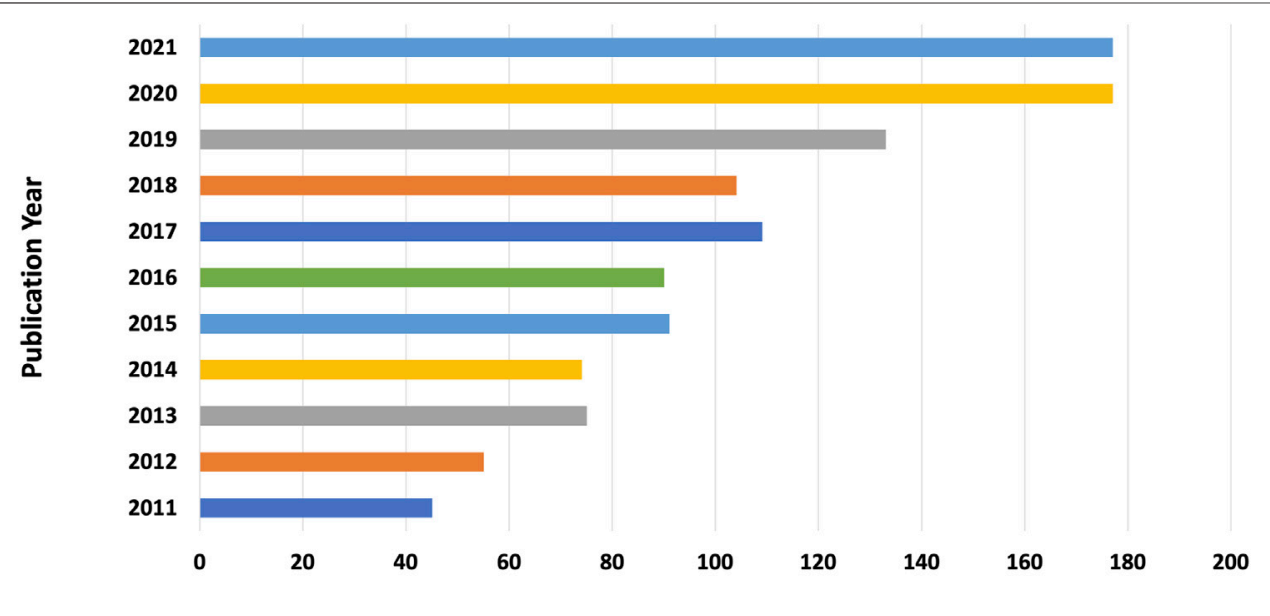

No. of publications

FIGURE 1 | Annual trend of publications. 
TABLE 1 | Search strategy.

\begin{tabular}{|c|c|}
\hline Set & Search query \\
\hline$\# 1$ & 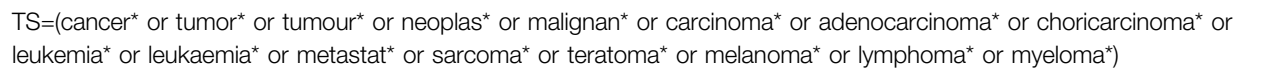 \\
\hline \#2 & $\begin{array}{l}\left\{\mathrm{TS}=\left[\text { physical }{ }^{\star} \text { near } / 5\left(\text { fit }^{*} \text { or activit }{ }^{\star} \text { or movement }{ }^{\star}\right)\right]\right\} \text { OR }\left[T S=\left(\text { exercis }^{\star} \text { or aerobic }{ }^{\star} \text { or walk }{ }^{*} \text { or endurance* or training or tai ji or }\right.\right. \\
\text { yoga or tai-chi or tai-ji or tai chi or taiji }{ }^{\star} \text { or pilates)] }\end{array}$ \\
\hline \#3 & (\#2) AND (\#1) \\
\hline \#4 & {$[\mathrm{TS}=($ molecular mechanism)] AND (\#3) } \\
\hline
\end{tabular}

A

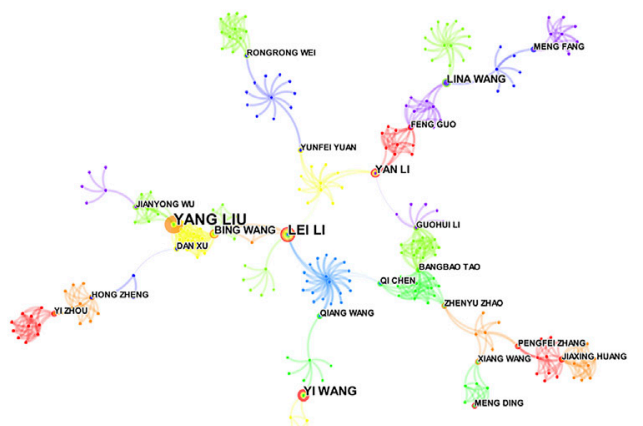

B

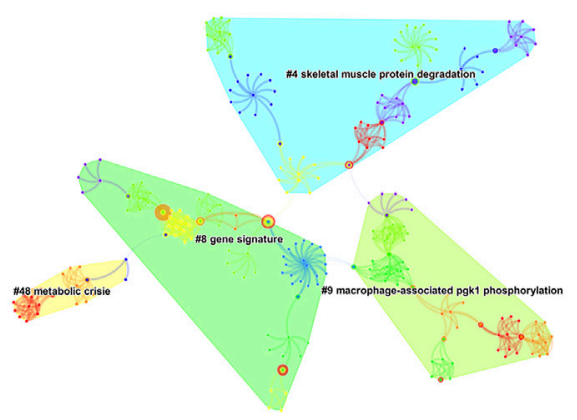

FIGURE 2 | (A) The network of co-authorship. (B) The network of co-authorship clusters.

\section{RESULTS}

\section{Distribution of Articles by Publication Years}

After removing 61 unqualified records, 1,130 articles were obtained. Figure 1 shows that the number of publications has generally increased with some fluctuations, ranging from 45 to 177 publications. From 2019 to 2020, the number of related publications increased the most (33 publications), indicating that more and more researchers are beginning to pay attention to this field.

\section{Co-Authorship, Co-Institution, and Co-Country}

We analyzed publications with time slicing of 1 year and the top 50 levels of most-cited or occurred items from each slice. Tree ring history was selected as node display pattern. Lines between nodes represent cooperation, and the color of lines indicates the first cooperation year.
7,588 nodes and 23,925 links composed of the merged coauthorship network, and we chose to visualize the largest connected component only (Figure 2A). The co-authorship network shows prolific authors and the collaboration among them. The most productive author was Lee $\mathrm{W}$ Jones with a total of 8 articles, followed by Jing Li (6 articles) and Yang Liu (6 articles). Although many authors have published relevant articles, there was little collaboration among them. Besides, the centrality of authors was relatively low, suggesting that more high-quality and large-scale collaborations are needed in the future.

In general, the silhouette value is used to evaluate the clusters. If the silhouette value is over 0.7 , the cluster is efficient and convincing. Four clusters (the silhouette value of 4 clusters exceeded over 0.9) were produced by log-likelihood ratio, mainly around skeletal muscle protein degradation, gene signature, metabolic crisis, macrophage-associated PGK-1 phosphorylation (Figure 2B). 
A

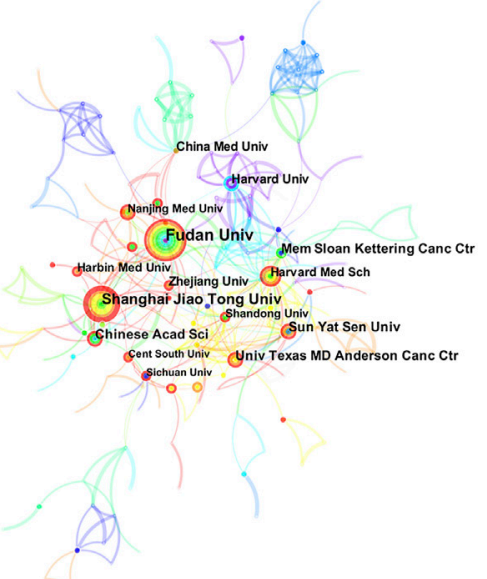

B

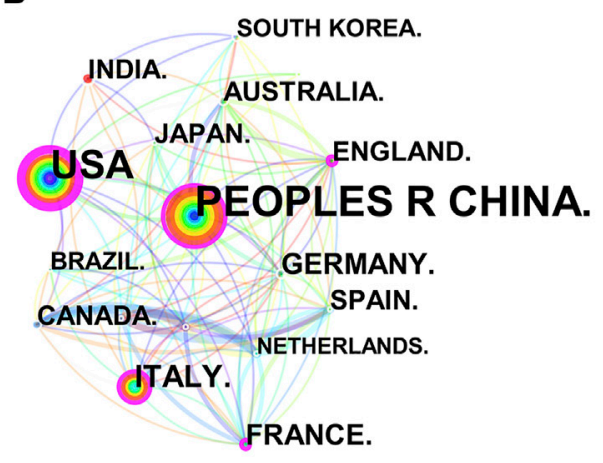

FIGURE 3 | (A) The network of co-institution. (B) The network of co-country.

A
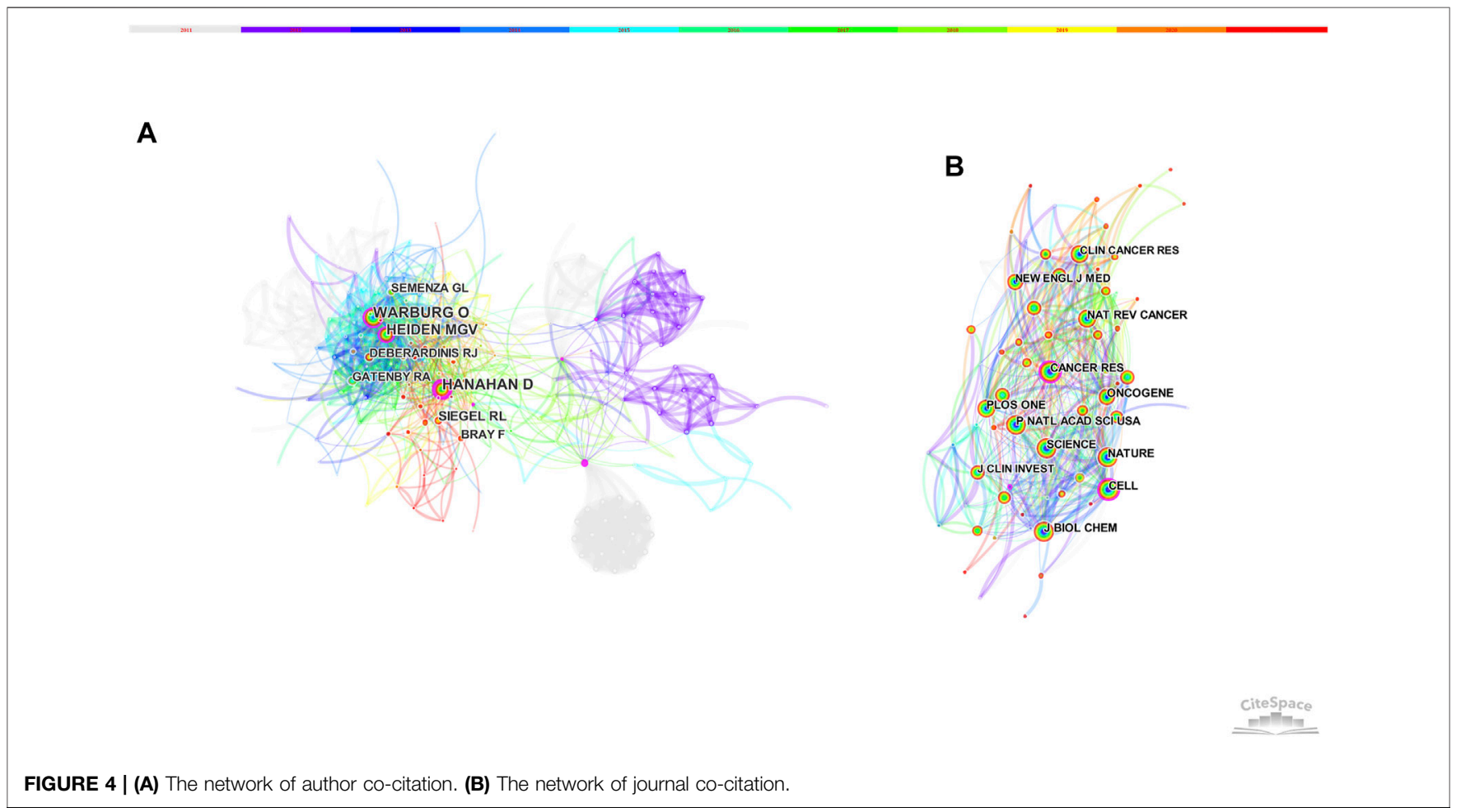

The merged co-institution network map is shown in Figure 3A, with 1,568 nodes and 5,227 links. The top five institutions were Fudan University (36 articles), Shanghai Jiao Tong University (36 articles), Sun Yat-sen University (22 articles), Chinese Academy of Sciences (21 articles) and Memorial Sloan Kettering Cancer Center (19 articles).

The merged co-country network consists of 86 nodes and 433 links. People's Republic of China was the leading country (427 
TABLE 2 | The top five cited journal.

\begin{tabular}{|c|c|c|c|c|c|c|}
\hline Rank & Frequency & Cited journal & IF & Centrality & Cited journal & IF \\
\hline 2 & 634 & Nature & 49.962 & 0.14 & Cell & 41.584 \\
\hline 3 & 629 & Cancer research & 12.701 & 0.1 & The biochemical journal & 3.857 \\
\hline 5 & 587 & Cell & 41.584 & 0.08 & International journal of cancer & 7.396 \\
\hline
\end{tabular}

A

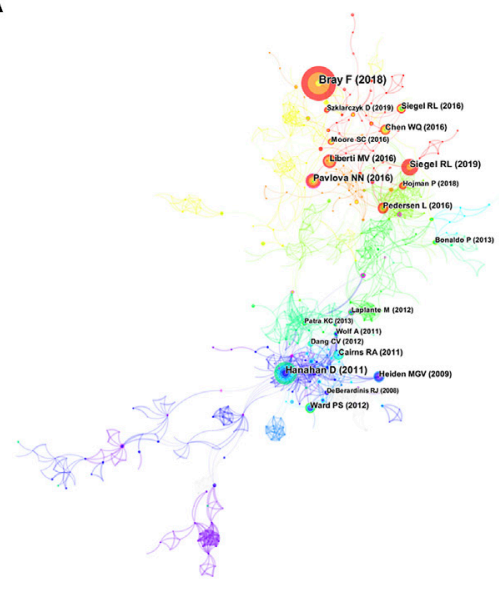

B

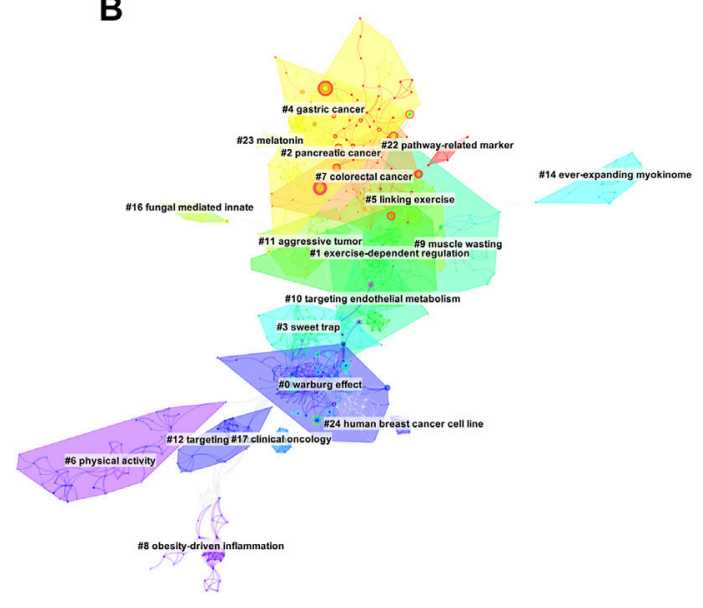

FIGURE 5 | (A) The network of co-cited references. (B) The network of co-cited references clusters.

articles), followed by United States (316 articles), Italy (87 articles), Germany (57 articles) and France (51 articles). Except for Germany, the betweenness centrality of other countries were both over 0.1, illustrating the important contribution of these countries in this area (Figure 3B).

\section{Author and Journal Co-Citation}

Figure 4 displays the network of author and journal co-citation. The most-cited authors were Hanahan D (130 citations) and Warburg O (130 citations), followed by Heiden MGV (119 citations), Siegel RL (74 citations), Semenza GL (62 citations) and Deberardinis RJ (61 citations). Besides, Hanahan D, Warburg $\mathrm{O}$ and Heiden MGV were also high-centrality authors (Figure 4A).

The top-ranked journal by citation counts was PLOS ONE with 638 citations, followed by Nature (634 citations), Cancer Research (629 citations), Proceedings of the National Academy of Sciences of the United States of America (627 citations) and Cell (587 citations) (Figure 4B). Besides, Cancer Research and Cell got both high frequency and high betweenness centrality, indicating its critical role in this field (Table 2).

\section{References Co-Citation}

Figure 5A and Table 3 show the top co-cited references with high frequency and high betweenness centrality. The first co-cited reference was published by Bray $\mathrm{F}$ et al., which provided a status report on the global burden of cancer (Bray et al., 2018), The reference published by Hanahan D introduced the hallmarks of cancer, which may affect the development of anti-cancer therapies (Hanahan and Weinberg, 2011). Siegel RL provided the cancer statistics of United States in 2019 (Siegel et al., 2019). Pavlova NN made a summary of the emerging 6 hallmarks of cancer metabolism (Pavlova and Thompson, 2016). Liberti MV summarized the explanations and controversies for the function of Warburg Effect (Liberti and Locasale, 2016).

Other high-cited publications were as follows: Pedersen $\mathrm{L}$ et al. revealed that exercise-induced muscle-derived interleukin-6 (IL-6) was involved in natural killer (NK) cell redistribution, thus reduced the incidence and growth of cancer (Pedersen et al., 2016). Hojman $P$ et al. concluded that the tumor growth-inhibitory effect of exercise was probably mediated by several different mechanisms (the cellular immune system and exercise-induced myokines) (Hojman et al., 
TABLE 3 | The top five cited references.

\begin{tabular}{|c|c|c|c|c|c|c|c|c|}
\hline Rank & $\begin{array}{c}\text { First } \\
\text { author }\end{array}$ & Country & Frequency & Centrality & Year & Cited references & Journal & IF \\
\hline 1 & Bray F & France & 53 & 0 & 2018 & $\begin{array}{l}\text { Global cancer statistics 2018: GLOBOCAN estimates of } \\
\text { incidence and mortality worldwide for } 36 \text { cancers in } 185 \\
\text { countries }\end{array}$ & $\begin{array}{l}\text { CA: a cancer journal } \\
\text { for clinicians }\end{array}$ & 508.702 \\
\hline 2 & Hanahan D & Switzerland & 35 & 0.06 & 2011 & Hallmarks of cancer: the next generation & Cell & 41.584 \\
\hline 3 & Siegel RL & United States & 26 & 0.01 & 2019 & Cancer statistics, 2019 & $\begin{array}{l}\text { CA: a cancer journal } \\
\text { for clinicians }\end{array}$ & 508.702 \\
\hline 4 & $\begin{array}{l}\text { Pavlova } \\
\text { NN }\end{array}$ & United States & 24 & 0.11 & 2016 & The emerging hallmarks of cancer metabolism & Cell Metabolism & 27.287 \\
\hline 5 & Liberti MV & United States & 21 & 0.02 & 2016 & The warburg effect: How does it benefit cancer cells? & $\begin{array}{l}\text { Trends in biochemical } \\
\text { sciences }\end{array}$ & 13.807 \\
\hline
\end{tabular}

2018). Moore SC et al. found that leisure-time physical activity was associated with lower risks of many cancers (Moore et al., 2016).

Figure 5B shows the largest 19 clusters of co-cited references. Figure 6 displays the top 41 references with strongest citation bursts, which indicating the emerging trends or increasing interests in the field. Generally, the most co-cited references usually got the strongest citation bursts.

\section{Keywords Co-Citation and Clusters}

Keywords are the high-level summary. High-frequency and highcentrality keywords often reflect hot research topics in this field. We analyzed publications with time slicing of 1 year and the top 30 levels of most-cited or occurred items from each slice. 149 nodes and 1,138 links composed of the merged co-occurring keywords network. Table 4 presents the top 10 keywords with high frequency and centrality. 6 clusters were produced by log-likelihood ratio, including metabolic syndrome, aerobic glycolysis, metabolic reprogramming, pharmacological suppression, molecular switch and atherosclerosis (Figure 7).

\section{Keywords With Citation Bursts}

Figure 8 presents the top 33 keywords with citation bursts. The blue line indicates the time interval, while the red line indicates the time period when a keyword had a burst (Chen et al., 2014). Keywords "warburg effect" with the strongest citation bursts appeared in 2014, indicating the importance of glucose metabolism in cancer cells. The most recent keywords with citation bursts occurred in 2019 were "inflammation", "hepatocellular carcinoma" and "adipose tissue". In addition, "epithelial mesenchymal transition" also continued to 2021.

\section{DISCUSSION}

\section{Summary of Findings}

A total of 1,130 related articles were retrieved eventually. In the past several years, increasing researchers have begun to study the molecular mechanisms of exercise on cancer, forming a group of prolific authors represented by Lee W Jones, Jing Li and Yang Liu. China was the leading country in this field and Chinese universities have published many relevant studies. Top-cited authors and references generally focused on the epidemiology and hallmarks of cancer. Top five keywords with both high frequency and high betweenness centrality were breast cancer, aerobic glycolysis, oxidative stress, gene expression, skeletal muscle. Keyword "warburg effect" ranked first with the highest citation burst, while "inflammation", "hepatocellular carcinoma", "epithelial mesenchymal transition", and "adipose tissue" were emerging research foci.

\section{Research Hotspots on Molecular Mechanisms of Exercise on Cancer}

Based on the results of CiteSpace, we summarized the hotresearch molecular mechanisms of exercise on cancer (Figure 9).

\section{Metabolism}

Aerobic Glycolytic/Warburg Effect

Altered glycolysis/TCA cycle has been proved to be one of hallmarks of cancer (Liberti and Locasale, 2016; Pavlova and Thompson, 2016). Highly up-regulated glycolytic phenotype could induce local acidosis, which is conducive to tumor development and invasion. Warburg effect is characterized by accelerated aerobic glycolytic metabolism even if there is sufficient oxygen supply, and is common in most malignant tumors (Cairns et al., 2011). In healthy athletes, increased systemic lactate levels during repeated high-intensity anaerobic exercise could inhibit glycolysis and net lactate production (Hollidge-Horvat et al., 1999). Evidence indicated that high-intensity anaerobic exercise was shown to inhibit the Warburg-type highly glycolytic (Hofmann, 2018). Animal studies also demonstrated that high-intensity anaerobic exercise training may have stronger effects on tumor growth compared with moderateintensity aerobic exercise (Bacurau et al., 2007; de Lima et al., 2008; Paceli et al., 2012). However, the correlation between the host and cancer metabolism is related to the duration, time, intensity, and movement mode of exercise (Koelwyn et al., 2017).

\section{Insulin/Insulin-Like Growth Factors}

Insulin and the insulin-like growth factors (IGFs) family play an essential role in regulating cell growth and apoptosis, as well as proliferation and differentiation of cancer cells (Hankinson et al., 1998; Christopoulos et al., 2015). Besides, insulin-like growth factor binding protein 3 (IGFBP-3) could inhibit cell proliferation and promote cell apoptosis by regulating local IGF-1 concentration and the anti-apoptosis effect of IGFBP-3 can be inhibited in breast cancer cells (Kim et al., 2004). Irwin et al. revealed that the decrease in IGF-I and IGFBP- 3 caused by exercise may explain the link between higher 


\section{Top 41 References with the Strongest Citation Bursts}

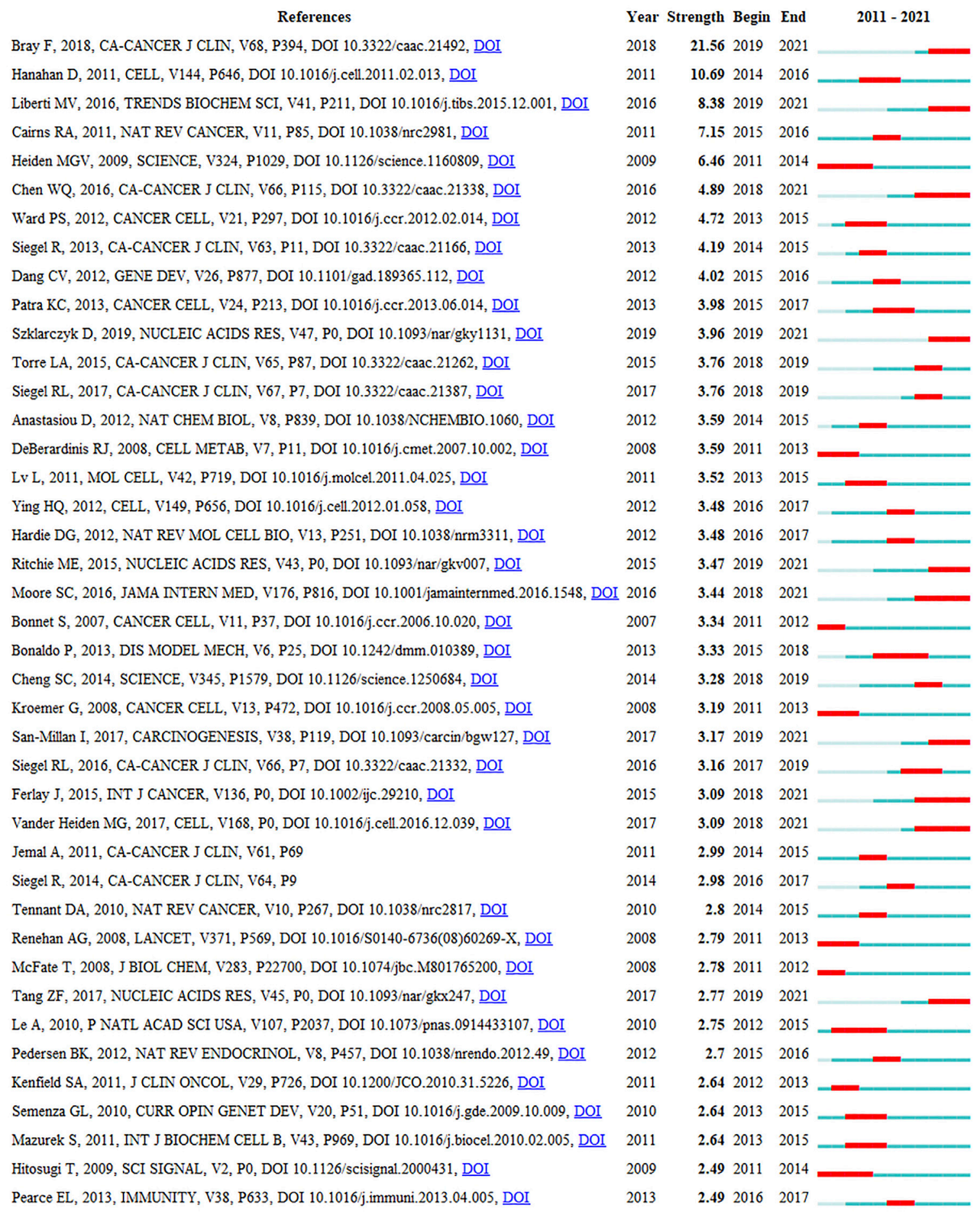

FIGURE 6 | Top 41 references with strongest citation bursts.

levels of physical activity and the survival rate of breast cancer patients (Irwin et al., 2009). Another study indicated that changes in insulin levels and/or changes in body fat or fat deposition by exercise may mediate breast cancer prognosis in part (Ligibel et al., 2008).

\section{Myokine/Apoptosis}

During exercise, paracrine factors coordinate tissue remodeling in response to skeletal muscle contraction. Factors secreted from muscle cells may influence cancer cell growth. Lack of physical activity 
TABLE 4 | Top 10 keywords in terms of frequency and centrality.

\begin{tabular}{|c|c|c|c|c|}
\hline Rank & Frequency & Keywords & Centrality & Keywords \\
\hline 2 & 113 & Aerobic glycolysis & 0.14 & Breast cancer \\
\hline 5 & 69 & Skeletal muscle & 0.12 & Skeletal muscle \\
\hline 6 & 69 & Metabolism & 0.07 & Colorectal cancer \\
\hline 7 & 45 & Colorectal cancer & 0.06 & Apoptosis \\
\hline 10 & 30 & Insulin resistance & 0.04 & Metabolism \\
\hline
\end{tabular}

A

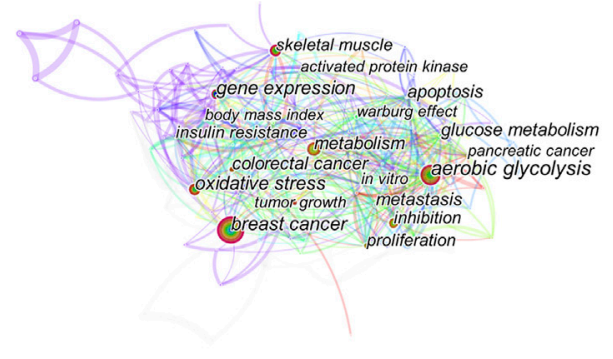

B

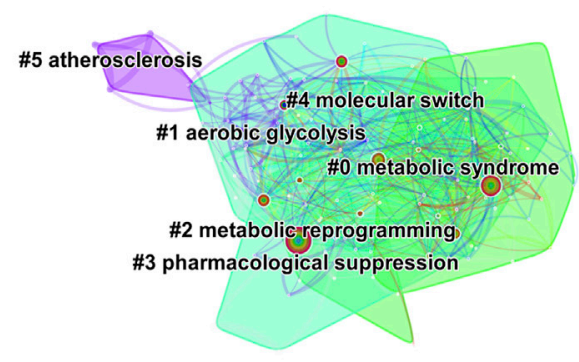

FIGURE 7 | (A) The network of co-occurring keywords. (B) The network of co-occurring keywords clusters.

probably leads to changes in myokine response. Evidence showed that myokines, such as the IL- 6 superfamily, may mediate some of the inhibitory effects of exercise on mammary cancer cell proliferation (Hojman et al., 2011). Moreover, exercise can induce apoptosis of tumor cells in skeletal muscle. DNA microarrays were used to compare the transcriptome of muscle tissue in young and old mice (sedentary and exercised), and the results showed that exercise was able to stimulate the secretion of secreted protein acidic and cysteinerich (SPARC) from muscle tissues and SPARC could inhibit colon tumorigenesis by increasing apoptosis (Aoi et al., 2013). Animal studies also suggested that moderate-intensity training may inhibit cancer cell proliferation and induce apoptosis (Westerlind et al., 2002).

\section{Oxidative Stress}

High levels of oxidative stress is another hallmark of cancer (Cairns et al., 2011), which is caused by the imbalance between the production and elimination of reactive oxygen species (ROS). Increased ROS levels are generally detrimental to cells, and could promote tumor formation via inducing DNA damage, proinflammatory cytokines (Naik and Dixit, 2011) and activating the nuclear factor- $\kappa \mathrm{B}(\mathrm{NF}-\kappa \mathrm{B})$ pathway (Gloire et al., 2006). Studies showed that exercise was able to improve antioxidation and counteract the negative consequences of oxidative stress by modulating systemic oxidative status (SOS) and DNA repair capability (Tomasello et al., 2017; Simioni et al., 2018). However, controversy exists since strenuous exercise may enhance oxidative stress.

\section{Cancer Types}

According to the high frequency keywords, breast cancer, colorectal cancer and hepatocellular carcinoma are main research cancer types of mechanisms of exercise. Possible underlying mechanisms of 


\section{Top 33 Keywords with the Strongest Citation Bursts}

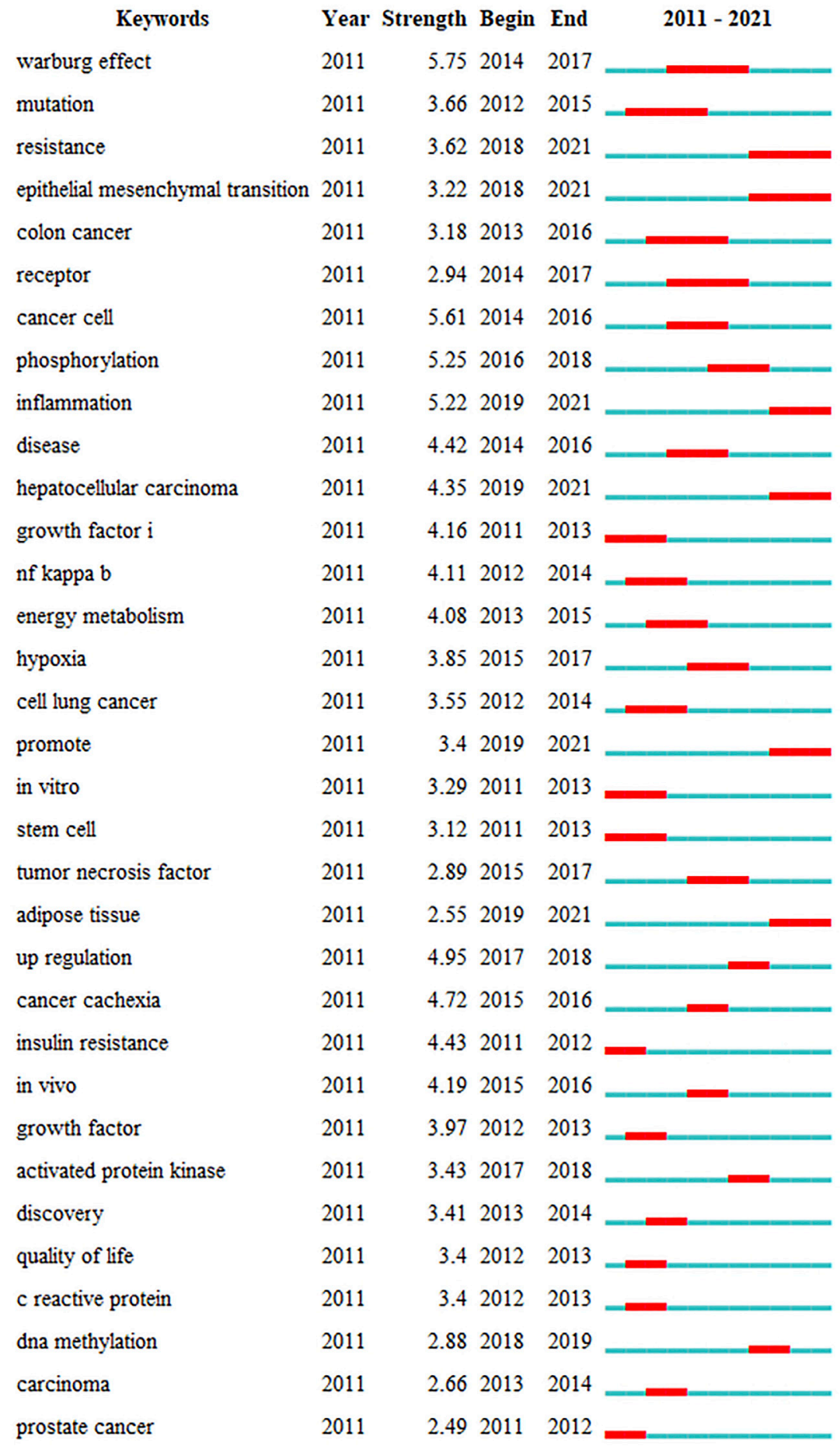

FIGURE 8 | Top 33 keywords with strongest citation bursts.

breast cancer includes a reduction of sex hormones, metabolic hormones, adipokines and oxidative stress, and an improvement of the immune function (de Boer et al., 2017). Hayes et al. also outlined the mechanisms of exercise for colorectal and prostate cancer through harnessing the immune system (Hayes et al., 2016; Song and Chan, 2018). Besides, hepatocellular carcinoma was one of the most recent keywords with citation bursts, which has attracted the attention of researchers. Exercise can attenuate the progression of 


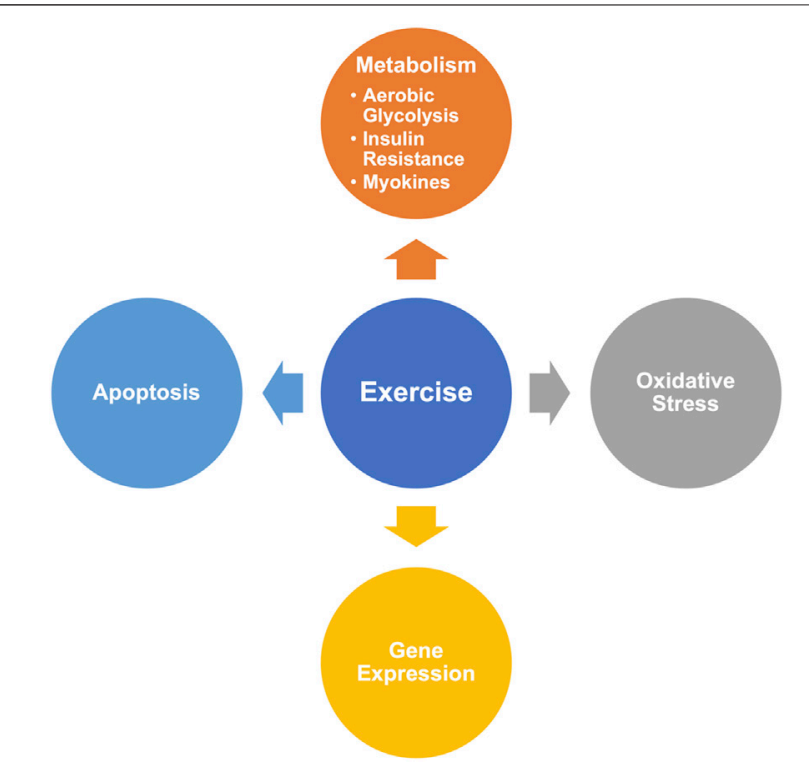

FIGURE 9 | Summary of the hot-research mechanisms.

hepatocellular carcinoma related to changes in key signaling pathways, cellular proliferation, tumor vascularization, and necrosis (Saran et al., 2018).

\section{Emerging Areas on Molecular Mechanisms of Exercise on Cancer}

From the citation bursts analysis, the most recent keywords with citation bursts occurred in 2019 was inflammation. Chronic inflammation is bound up with the development and progression of cancer (Gleeson et al., 2011; Friedenreich et al., 2012). Numerous molecules such as tumor necrosis factor (TNF)- $\alpha$, interleukin-1 (IL1) and IL-6 are common biomarkers of inflammation associated with cancer, and they could be regulated by the transcription factor NF-kB (Aggarwal et al., 2009). Khosravi et al. has proved the antiinflammatory effects of exercise, especially in prostate and breast cancer survivors (Khosravi et al., 2019). Besides, recent studies suggested that obesity related-excess adipose tissue could promote the neoplasia and progression of tumor through adipose tissue inflammation (circulating cytokines such as TNF- $\alpha$ and IL-6) (Campbell et al., 2009; Iyengar et al., 2013). Accompanied by weight loss, exercise could attenuate adipose tissue inflammation in aged 20-40 years, overweight men (Auerbach et al., 2013; Ahmadizad et al., 2014) and obese postmenopausal breast cancer survivors (Dieli-Conwright et al., 2018).

The NF- $\mathrm{BB}$ family is composed of transcription factors and plays a complex and key role in the regulation of immune responses and inflammation (Tilborghs et al., 2017). NF- $\mathrm{kB}$ could be activated by

\section{REFERENCES}

Aggarwal, B. B., Vijayalekshmi, R. V., and Sung, B. (2009). Targeting Inflammatory Pathways for Prevention and Therapy of Cancer: Short-Term Friend, Long- almost all infectious agents links with cancer, e.g. human papillomavirus (James et al., 2006), HIV(DeLuca et al., 1996) and Helicobacter pylori (Keates et al., 1997). It was proved that exercise training could prevent tumor-induced TWEAK/NF- $\kappa \mathrm{B}$ signaling pathway in skeletal muscle and had a beneficial effect on fiber cross-sectional area and metabolism. This exercise-induced muscle remodeling was related to less malignant mammary lesions in tumor-bearing animals (Padrao et al., 2017).

These findings provide new insights into the potential anti-cancer role of exercise. Since exercise has different parameters, future studies could carry in-depth research of interactions between different mechanisms and try to elucidate the recommended doses and intensities of exercise for cancer, especially in breast, colorectal, prostate cancer and hepatocellular carcinoma.

Compared with other reviews, our study based on Citespace provided a visualized research hotspots and frontiers. However, there are still some limitations in this study. Due to the limitation of Citespace software, we only searched SCI-Expanded and analyzed studies published in English. Therefore, the data may not be comprehensive enough. Articles published in other languages and databases need further research.

\section{CONCLUSION}

This study analyzed the research hot spots and frontiers of molecular mechanisms of exercise on cancer via CiteSpace. Based on the results, altered metabolism (aerobic glycolysis, insulin resistance, myokines), oxidative stress, gene expression and apoptosis were hotresearch mechanisms of exercise on cancer. Emerging research foci of mechanisms were generally around inflammation, epithelial mesenchymal transition and adipokines. In addition, future studies could carry in-depth research of interactions between different mechanisms and try to elucidate the recommended doses and intensities of exercise for cancer, especially in breast, colorectal, prostate cancer and hepatocellular carcinoma.

\section{AUTHOR CONTRIBUTIONS}

RJ and JL designed the study. DZ and YL drafted the manuscript. $\mathrm{XH}$ revised the manuscript. $\mathrm{YH}$ analyzed the data. All authors contributed to the article and approved the submitted version.

\section{FUNDING}

The National Natural Science Foundation of China (Grant Numbers 81873356 and 82104976). The National Key Research and Development Project of China (Grant Number 2019YFC1710302).

Term Foe. Clin. Cancer Res. 15 (2), 425-430. doi:10.1158/1078-0432.CCR-080149

Ahmadizad, S., Ghorbani, S., Ghasemikaram, M., and Bahmanzadeh, M. (2014). Effects of Short-Term Nonperiodized, Linear Periodized and Daily Undulating Periodized Resistance Training on Plasma Adiponectin, Leptin and Insulin 
Resistance. Clin. Biochem. 47 (6), 417-422. doi:10.1016/ j.clinbiochem.2013.12.019

Aoi, W., Naito, Y., Takagi, T., Tanimura, Y., Takanami, Y., Kawai, Y., et al. (2013). A Novel Myokine, Secreted Protein Acidic and Rich in Cysteine (SPARC), Suppresses colon Tumorigenesis via Regular Exercise. Gut 62 (6), 882-889. doi:10.1136/gutjnl-2011-300776

Auerbach, P., Nordby, P., Bendtsen, L. Q., Mehlsen, J. L., Basnet, S. K., Vestergaard, H., et al. (2013). Differential Effects of Endurance Training and Weight Loss on Plasma Adiponectin Multimers and Adipose Tissue Macrophages in Younger, Moderately Overweight Men. Am. J. Physiology-Regulatory, Integr. Comp. Physiol. 305 (5), R490-R498. doi:10.1152/ajpregu.00575.2012

Bacurau, A. V. N., Belmonte, M. A., Navarro, F., Moraes, M. R., Pontes, F. L., Pesquero, J. L., et al. (2007). Effect of a High-Intensity Exercise Training on the Metabolism and Function of Macrophages and Lymphocytes of Walker 256 Tumor-Bearing Rats. Exp. Biol. Med. (Maywood) 232, 1289-1299. doi:10.3181/0704-RM-93

Bray, F., Ferlay, J., Soerjomataram, I., Siegel, R. L., Torre, L. A., and Jemal, A. (2018). Global Cancer Statistics 2018: GLOBOCAN Estimates of Incidence and Mortality Worldwide for 36 Cancers in 185 Countries. CA: A Cancer J. Clinicians 68 (6), 394-424. doi:10.3322/caac.21492

Cairns, R. A., Harris, I. S., and Mak, T. W. (2011). Regulation of Cancer Cell Metabolism. Nat. Rev. Cancer 11 (2), 85-95. doi:10.1038/nrc2981

Campbell, K. L., Makar, K. W., Kratz, M., Foster-Schubert, K. E., McTiernan, A., and Ulrich, C. M. (2009). A Pilot Study of Sampling Subcutaneous Adipose Tissue to Examine Biomarkers of Cancer Risk. Cancer Prev. Res. 2 (1), 37-42. doi:10.1158/1940-6207.CAPR-08-0073

Campbell, K. L., Winters-Stone, K. M., Wiskemann, J., May, A. M., Schwartz, A. L., Courneya, K. S., et al. (2019). Exercise Guidelines for Cancer Survivors: Consensus Statement from International Multidisciplinary Roundtable. Med. Sci. Sports Exerc. 51 (11), 2375-2390. doi:10.1249/MSS.0000000000002116

Chen, C. (2006). CiteSpace II: Detecting and Visualizing Emerging Trends and Transient Patterns in Scientific Literature. J. Am. Soc. Inf. Sci. 57 (3), 359-377. doi:10.1002/asi.20317

Chen, C., Dubin, R., and Kim, M. C. (2014). Emerging Trends and New Developments in Regenerative Medicine: a Scientometric Update (2000 - 2014). Expert Opin. Biol. Ther. 14 (9), 1295-1317. doi:10.1517/14712598.2014.920813

Chen, C. (2004). Searching for Intellectual Turning Points: Progressive Knowledge Domain Visualization. Proc. Natl. Acad. Sci. 101 (Suppl. 1), 5303-5310. doi:10.1073/pnas.0307513100

Christopoulos, P. F., Msaouel, P., and Koutsilieris, M. (2015). The Role of the Insulin-like Growth Factor-1 System in Breast Cancer. Mol. Cancer 14, 43. doi:10.1186/s12943-015-0291-7

de Boer, M. C., Wörner, E. A., Verlaan, D., and van Leeuwen, P. A. M. (2017). The Mechanisms and Effects of Physical Activity on Breast Cancer. Clin. Breast Cancer 17 (4), 272-278. doi:10.1016/j.clbc.2017.01.006

de Lima, C., Alves, L. E., Iagher, F., Machado, A. F., Bonatto, S. J., Kuczera, D., et al. (2008). Anaerobic Exercise Reduces Tumor Growth, Cancer Cachexia and Increases Macrophage and Lymphocyte Response in Walker 256 TumorBearing Rats. Eur. J. Appl. Physiol. 104 (6), 957-964. doi:10.1007/s00421008-0849-9

DeLuca, C., Roulston, A., Koromilas, A., Wainberg, M. A., and Hiscott, J. (1996). Chronic Human Immunodeficiency Virus Type 1 Infection of Myeloid Cells Disrupts the Autoregulatory Control of the NF-kappaB/Rel Pathway via Enhanced IkappaBalpha Degradation. J. Virol. 70 (8), 5183-5193. doi:10.1128/JVI.70.8.5183-5193.1996

Dieli-Conwright, C. M., Parmentier, J.-H., Sami, N., Lee, K., Spicer, D., Mack, W. J., et al. (2018). Adipose Tissue Inflammation in Breast Cancer Survivors: Effects of a 16-week Combined Aerobic and Resistance Exercise Training Intervention. Breast Cancer Res. Treat. 168 (1), 147-157. doi:10.1007/s10549-017-4576-y

Friedenreich, C. M., Neilson, H. K., Woolcott, C. G., Wang, Q., Stanczyk, F. Z., McTiernan, A., et al. (2012). Inflammatory Marker Changes in a Yearlong Randomized Exercise Intervention Trial Among Postmenopausal Women. Cancer Prev. Res. 5 (1), 98-108. doi:10.1158/1940-6207.CAPR-11-0369

Galvão, D. A., Taaffe, D. R., Spry, N., Joseph, D., and Newton, R. U. (2010). Combined Resistance and Aerobic Exercise Program Reverses Muscle Loss in Men Undergoing Androgen Suppression Therapy for Prostate Cancer without Bone Metastases: a Randomized Controlled Trial. Jco 28 (2), 340-347. doi:10.1200/JCO.2009.23.2488
Gleeson, M., Bishop, N. C., Stensel, D. J., Lindley, M. R., Mastana, S. S., and Nimmo, M. A. (2011). The Anti-inflammatory Effects of Exercise: Mechanisms and Implications for the Prevention and Treatment of Disease. Nat. Rev. Immunol. 11 (9), 607-615. doi:10.1038/nri3041

Gloire, G., Legrand-Poels, S., and Piette, J. (2006). NF-кB Activation by Reactive Oxygen Species: Fifteen Years Later. Biochem. Pharmacol. 72 (11), 1493-1505. doi:10.1016/j.bcp.2006.04.011

Hanahan, D., and Weinberg, R. A. (2011). Hallmarks of Cancer: the Next Generation. Cell 144 (5), 646-674. doi:10.1016/j.cell.2011.02.013

Hankinson, S. E., Willett, W. C., Colditz, G. A., Hunter, D. J., Michaud, D. S., Deroo, B., et al. (1998). Circulating Concentrations of Insulin-like Growth Factor I and Risk of Breast Cancer. The Lancet 351 (9113), 1393-1396. doi:10.1016/S0140-6736(97)10384-1

Hayes, B. D., Brady, L., Pollak, M., and Finn, S. P. (2016). Exercise and Prostate Cancer: Evidence and Proposed Mechanisms for Disease Modification. Cancer Epidemiol. Biomarkers Prev. 25 (9), 1281-1288. doi:10.1158/1055-9965.EPI-160223

Hayes, S. C., Newton, R. U., Spence, R. R., and Galvão, D. A. (2019). The Exercise and Sports Science Australia Position Statement: Exercise Medicine in Cancer Management. J. Sci. Med. Sport 22 (11), 1175-1199. doi:10.1016/ j.jsams.2019.05.003

Hofmann, P. (2018). Cancer and Exercise: Warburg Hypothesis, Tumour Metabolism and High-Intensity Anaerobic Exercise. Sports 6 (1), 10. doi:10.3390/sports6010010

Hojman, P., Dethlefsen, C., Brandt, C., Hansen, J., Pedersen, L., and Pedersen, B. K. (2011). Exercise-induced Muscle-Derived Cytokines Inhibit Mammary Cancer Cell Growth. Am. J. Physiology-Endocrinology Metab. 301 (3), E504-E510. doi:10.1152/ajpendo.00520.2010

Hojman, P., Gehl, J., Christensen, J. F., and Pedersen, B. K. (2018). Molecular Mechanisms Linking Exercise to Cancer Prevention and Treatment. Cel Metab. 27 (1), 10-21. doi:10.1016/j.cmet.2017.09.015

Hollidge-Horvat, M. G., Parolin, M. L., Wong, D., Jones, N. L., and Heigenhauser, G. J. F. (1999). Effect of Induced Metabolic Acidosis on Human Skeletal Muscle Metabolism during Exercise. Am. J. Physiology-Endocrinology Metab. 277 (4), E647-E658. doi:10.1152/ajpendo.1999.277.4.E647

Irwin, M. L., Varma, K., Alvarez-Reeves, M., Cadmus, L., Wiley, A., Chung, G. G., et al. (2009). Randomized Controlled Trial of Aerobic Exercise on Insulin and Insulin-like Growth Factors in Breast Cancer Survivors: the Yale Exercise and Survivorship Study. Cancer Epidemiol. Biomarkers Prev. 18 (1), 306-313. doi:10.1158/1055-9965.EPI-08-0531

Iyengar, N. M., Hudis, C. A., and Dannenberg, A. J. (2013). Obesity and Inflammation: New Insights into Breast Cancer Development and Progression. Am. Soc. Clin. Oncol. Educ. Book 33, 46-51. doi:10.14694/ edbook_am.2013.33.46

James, M. A., Lee, J. H., and Klingelhutz, A. J. (2006). Human Papillomavirus Type 16 E6 Activates NF-Kb, Induces cIAP-2 Expression, and Protects against Apoptosis in a PDZ Binding Motif-dependent Manner. J. Virol. 80 (11), 5301-5307. doi:10.1128/JVI.01942-05

Keates, S., Hitti, Y., Upton, M., and Kelly, C. (1997). Helicobacter pylori Infection Activates NF-Kappa B in Gastric Epithelial Cells. Gastroenterology 113 (4), 1099-1109. doi:10.1053/gast.1997.v113.pm9322504

Khosravi, N., Stoner, L., Farajivafa, V., and Hanson, E. D. (2019). Exercise Training, Circulating Cytokine Levels and Immune Function in Cancer Survivors: A Meta-Analysis. Brain Behav. Immun. 81, 92-104. doi:10.1016/ j.bbi.2019.08.187

Kim, H.-S., Ingermann, A. R., Tsubaki, J., Twigg, S. M., Walker, G. E., and Oh, Y. (2004). Insulin-like Growth Factor-Binding Protein 3 Induces Caspasedependent Apoptosis through a Death Receptor-Mediated Pathway in MCF7 Human Breast Cancer Cells. Cancer Res. 64 (6), 2229-2237. doi:10.1158/ 0008-5472.can-03-1675

Koelwyn, G. J., Quail, D. F., Zhang, X., White, R. M., and Jones, L. W. (2017). Exercise-dependent Regulation of the Tumour Microenvironment. Nat. Rev. Cancer 17 (10), 620-632. doi:10.1038/nrc.2017.78

Kyu, H. H., Bachman, V. F., Alexander, L. T., Mumford, J. E., Afshin, A., Estep, K., et al. (2016). Physical Activity and Risk of Breast Cancer, colon Cancer, Diabetes, Ischemic Heart Disease, and Ischemic Stroke Events: Systematic Review and Dose-Response Meta-Analysis for the Global Burden of Disease Study 2013. BMJ 354, i3857. doi:10.1136/bmj.i3857 
Liberti, M. V., and Locasale, J. W. (2016). The Warburg Effect: How Does it Benefit Cancer Cells? Trends Biochem. Sci. 41 (3), 211-218. doi:10.1016/j.tibs.2015.12.001

Ligibel, J. A., Campbell, N., Partridge, A., Chen, W. Y., Salinardi, T., Chen, H., et al. (2008). Impact of a Mixed Strength and Endurance Exercise Intervention on Insulin Levels in Breast Cancer Survivors. Jco 26 (6), 907-912. doi:10.1200/ JCO.2007.12.7357

Magné, N., Melis, A., Chargari, C., Castadot, P., Guichard, J.-B., Barani, D., et al. (2011). Recommendations for a Lifestyle Which Could Prevent Breast Cancer and its Relapse: Physical Activity and Dietetic Aspects. Crit. Rev. Oncology/ Hematology 80 (3), 450-459. doi:10.1016/j.critrevonc.2011.01.013

Metsios, G. S., Moe, R. H., and Kitas, G. D. (2020). Exercise and Inflammation. Best Pract. Res. Clin. Rheumatol. 34 (2), 101504. doi:10.1016/j.berh.2020.101504

Moore, S. C., Lee, I.-M., Weiderpass, E., Campbell, P. T., Sampson, J. N., Kitahara, C. M., et al. (2016). Association of Leisure-Time Physical Activity with Risk of 26 Types of Cancer in 1.44 Million Adults. JAMA Intern. Med. 176 (6), 816-825. doi:10.1001/jamainternmed.2016.1548

Naik, E., and Dixit, V. M. (2011). Mitochondrial Reactive Oxygen Species Drive Proinflammatory Cytokine Production. J. Exp. Med. 208 (3), 417-420. doi:10.1084/jem.20110367

Paceli, R. B., Cal, R. N., dos Santos, C. H. F., Cordeiro, J. A., Neiva, C. M., Nagamine, K. K., et al. (2012). The Influence of Physical Activity in the Progression of Experimental Lung Cancer in Mice. Pathol. - Res. Pract. 208 (7), 377-381. doi:10.1016/j.prp.2012.04.006

Padrão, A. I., Figueira, A. C. C., Faustino-Rocha, A. I., Gama, A., Loureiro, M. M., Neuparth, M. J., et al. (2017). Long-term Exercise Training Prevents Mammary Tumorigenesis-Induced Muscle Wasting in Rats through the Regulation of TWEAK Signalling. Acta Physiol. 219 (4), 803-813. doi:10.1111/apha.12721

Pavlova, N. N., and Thompson, C. B. (2016). The Emerging Hallmarks of Cancer Metabolism. Cel Metab. 23 (1), 27-47. doi:10.1016/j.cmet.2015.12.006

Pedersen, L., Idorn, M., Olofsson, G. H., Lauenborg, B., Nookaew, I., Hansen, R. H., et al. (2016). Voluntary Running Suppresses Tumor Growth through Epinephrine- and IL-6-Dependent NK Cell Mobilization and Redistribution. Cel Metab. 23 (3), 554-562. doi:10.1016/j.cmet.2016.01.011

Rock, C. L., Doyle, C., Demark-Wahnefried, W., Meyerhardt, J., Courneya, K. S., Schwartz, A. L., et al. (2012). Nutrition and Physical Activity Guidelines for Cancer Survivors. CA: A Cancer J. Clinicians 62 (4), 242-274. doi:10.3322/ caac. 21142

Saran, U., Guarino, M., Rodríguez, S., Simillion, C., Montani, M., Foti, M., et al. (2018). Anti-tumoral Effects of Exercise on Hepatocellular Carcinoma Growth. Hepatol. Commun. 2 (5), 607-620. doi:10.1002/hep4.1159

Schmitz, K. H., Courneya, K. S., Matthews, C., Demark-Wahnefried, W., Galvão, D. A., Pinto, B. M., et al. (2010). American College of Sports Medicine Roundtable on Exercise Guidelines for Cancer Survivors. Med. Sci. Sports Exerc. 42 (7), 1409-1426. doi:10.1249/MSS.0b013e3181e0c112

Siegel, R. L., Miller, K. D., and Jemal, A. (2019). Cancer Statistics, 2019. CA A. Cancer J. Clin. 69 (1), 7-34. doi:10.3322/caac.21551
Simioni, C., Zauli, G., Martelli, A. M., Vitale, M., Sacchetti, G., Gonelli, A., et al. (2018). Oxidative Stress: Role of Physical Exercise and Antioxidant Nutraceuticals in Adulthood and Aging. Oncotarget 9 (24), 17181-17198. doi:10.18632/oncotarget.24729

Song, M., and Chan, A. T. (2018). The Potential Role of Exercise and Nutrition in Harnessing the Immune System to Improve Colorectal Cancer Survival. Gastroenterology 155 (3), 596-600. doi:10.1053/j.gastro.2018.07.038

Speck, R. M., Courneya, K. S., Mâsse, L. C., Duval, S., and Schmitz, K. H. (2010). An Update of Controlled Physical Activity Trials in Cancer Survivors: a Systematic Review and Meta-Analysis. J. Cancer Surviv 4 (2), 87-100. doi:10.1007/s11764009-0110-5

Swift, D. L., McGee, J. E., Earnest, C. P., Carlisle, E., Nygard, M., and Johannsen, N. M. (2018). The Effects of Exercise and Physical Activity on Weight Loss and Maintenance. Prog. Cardiovasc. Dis. 61 (2), 206-213. doi:10.1016/ j.pcad.2018.07.014

Tilborghs, S., Corthouts, J., Verhoeven, Y., Arias, D., Rolfo, C., Trinh, X. B., et al. (2017). The Role of Nuclear Factor-Kappa B Signaling in Human Cervical Cancer. Crit. Rev. Oncology/Hematology 120, 141-150. doi:10.1016/ j.critrevonc.2017.11.001

Tomasello, B., Malfa, G. A., Strazzanti, A., Gangi, S., Di Giacomo, C., Basile, F., et al. (2017). Effects of Physical Activity on Systemic Oxidative/DNA Status in Breast Cancer Survivors. Oncol. Lett. 13 (1), 441-448. doi:10.3892/ol.2016.5449

Westerlind, K. C., McCarty, H. L., Gibson, K. J., and Strange, R. (2002). Effect of Exercise on the Rat Mammary Gland: Implications for Carcinogenesis. Acta Physiol. Scand. 175 (2), 147-156. doi:10.1046/j.1365-201X.2002.00980.x

Wolin, K. Y., Yan, Y., Colditz, G. A., and Lee, I.-M. (2009). Physical Activity and colon Cancer Prevention: a Meta-Analysis. Br. J. Cancer 100 (4), 611-616. doi:10.1038/sj.bjc.6604917

Conflict of Interest: The authors declare that the research was conducted in the absence of any commercial or financial relationships that could be construed as a potential conflict of interest.

Publisher's Note: All claims expressed in this article are solely those of the authors and do not necessarily represent those of their affiliated organizations, or those of the publisher, the editors and the reviewers. Any product that may be evaluated in this article, or claim that may be made by its manufacturer, is not guaranteed or endorsed by the publisher.

Copyright (C) 2022 Zhong, Li, Huang, Hong, Li and Jin. This is an open-access article distributed under the terms of the Creative Commons Attribution License (CC BY). The use, distribution or reproduction in other forums is permitted, provided the original author(s) and the copyright owner(s) are credited and that the original publication in this journal is cited, in accordance with accepted academic practice. No use, distribution or reproduction is permitted which does not comply with these terms. 